\title{
An overview of the dialogue meetings initiated by ICRP in Japan after the Fukushima accident
}

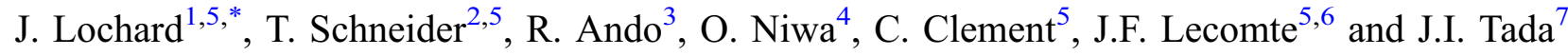 \\ ${ }^{1}$ Nagasaki University, Atomic Bomb Disease Institute, Nagasaki, Japan. \\ ${ }^{2}$ Nuclear Protection Evaluation Centre (CEPN), Fontenay-aux-Roses, France. \\ ${ }^{3}$ Ethos in Fukushima, Tabito, Japan. \\ ${ }^{4}$ Radiation Effects Research Foundation (RERF), Hiroshima, Japan. \\ 5 International Commission on Radiological Protection (ICRP), Ottawa, Canada, COUNTRY. \\ ${ }^{6}$ Institute for Radiological Protection and Nuclear Safety (IRSN), Fontenay-aux-Roses, France. \\ ${ }^{7}$ Radiation Safety Forum - Japan (RSFJ), Fukushima City, Japan.
}

Received: 30 April 2019 / Accepted: 6 May 2019

\begin{abstract}
In its recommendations for the protection of people living in long-term contaminated territories after a nuclear accident, the International Commission on Radiological Protection (ICRP) emphasizes the effectiveness of directly involving the affected population and local professionals in the management of the situation, and the responsibility of authorities at both national and local levels to create the conditions and provide the means favouring the involvement and empowerment of the population. In this perspective, ICRP initiated in the fall 2011 a dialogue between representatives of the Fukushima Prefecture, local professionals, local communities and representatives of Belarusian, Norwegian and French organisations to find ways to respond to the challenges of the long-term rehabilitation of the living conditions in the territories affected by the Fukushima nuclear power plant. After a first series of twelve "ICRP Dialogue" meetings between 2011 and 2015 closed by an international workshop, a group of local stakeholders took over and organized a new series of eight "Fukushima Dialogue" meetings between 2016 and 2018. The article gives an overview of the dialogue meetings initiated by ICRP in Japan after the Fukushima nuclear accident.
\end{abstract}

Keywords: Fukushima / dialogue / nuclear accident / nuclear emergency preparedness / radiation protection

\section{Introduction: the origin of the dialogue meetings}

Despite the fact that it is not an emergency response organisation, the International Commission on Radiological Protection (ICRP) strongly mobilized after the Fukushima accident. Beyond publicly expressing its sympathy to the Japanese population, it responded in the months following the accident to numerous requests for advice from authorities and experts directly involved in the management of the accident on how to implement the ICRP recommendations. Among the latter was the importance of involving stakeholders, which featured prominently in the ICRP publications 109 and 111 on the protection of people respectively during the emergency phase and the long-term phase after a nuclear accident (ICRP, 2009a, 2009 b). The last publication was largely based on the innovative experience of the Ethos Project for engaging stakeholders in the rehabilitation of living conditions in the context of the post-

\footnotetext{
*Corresponding author: lochard@nagasaki-u.ac.jp
}

Chernobyl accident. This project had taken place in the Stolyn district in southern Belarus in the late nineties (1996-2001) with the support of the European Commission and had been extended to 3 other districts in the framework of the CORE Programme supported by the Belarusian authorities and several international organisations from 2004 to 2008 (Hériard Dubreuil et al., 1999; Lochard, 2013).

It is in this context that at the request of $\mathrm{O}$. Niwa, then a member of the ICRP Main Commission, a mission was organized by J. Lochard and the Scientific Secretary of ICRP, C. Clement, in Belarus in October 11 in order to meet stakeholders in the affected territories and realized first-hand the challenges posed by the management of the long-term consequences of a nuclear accident. During this mission, in addition to meetings with Belarusian authorities and experts, it was an opportunity to have in-depth exchanges with residents, professionals and authorities of the Bragin District adjacent to the $30 \mathrm{~km}$ forbidden zone around the Chernobyl nuclear power plant, who participated actively in the CORE Programme.

Inspired by what he had learned in Belarus, O. Niwa, newly appointed as special professor at Fukushima Medical 


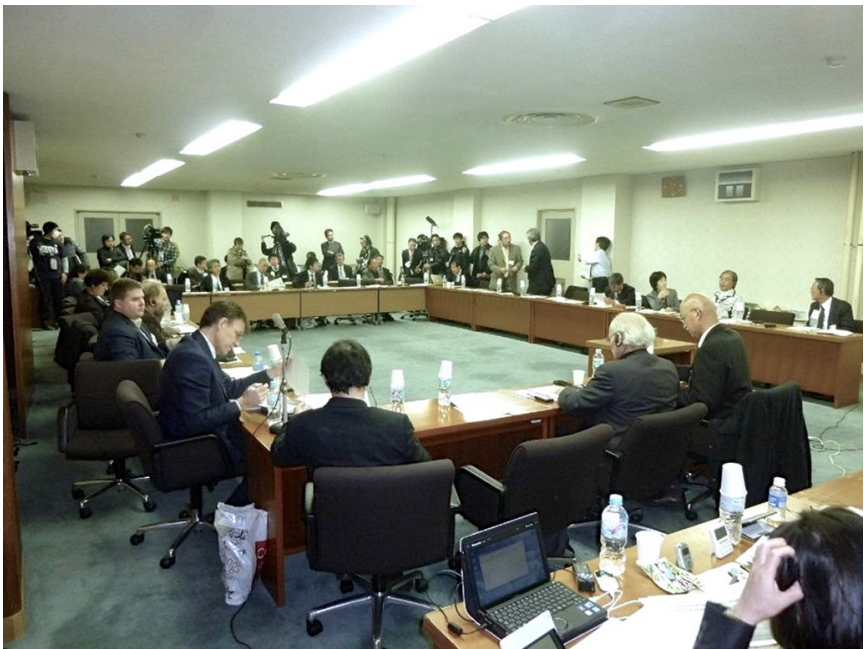

Fig. 1. The first dialogue meeting in Fukushima in November 11.

University (FMU) and directly confronted to the difficult situation at that time in Japan, together with C. Clement decided to organize a dialogue meeting in Fukushima City in November 11 with the support of J.I. Tada from the Radiation Safety Forum Japan (RSFJ), a non-profit organization (NPO) involved in the first decontamination actions together with affected residents. The purpose of this dialogue was to engage discussions between local stakeholders to reflect together on ways to respond to the numerous challenges they were facing in the territories affected by the accident. Although it was unsure initially if further meetings would be held, after listening to two days of intense discussions, the organizers felt that the dialogue should continue and it turns out that 19 other meetings followed up to the end of 2018.

The present article describes the dynamics and the key features and results of this series of meetings and then draws some of the main lessons learnt through these dialogues for the management of the recovery process after a large nuclear accident.

\section{The ICRP Dialogue Initiative (2011-2015)}

\subsection{The first dialogue meeting and the connection with Ethos in Fukushima}

The first dialogue meeting was held on 26-27 November 11 at the Prefectural Council Hall in Fukushima City under the title: "The rehabilitation of living conditions after the Fukushima accident: lessons from Chernobyl and ICRP Recommendations". It was organized by ICRP in cooperation with the Radiation Safety Forum Japan (NPO), the Fukushima Prefecture and FMU. Participants (Fig. 1) from the Fukushima Prefecture and national authorities, representatives of Date, Kawauchi and litate, professionals from affected localities, scientists from universities and national institutes, local and national media, national and international NPOs, representatives of ICRP and of the Committee on Radiological Protection and Public Health of the OECD Nuclear Energy Agency were present. Experts from Belarus, Norway and France with direct experience in managing long-term consequences of the Chernobyl accident attended the meeting and made presentations.
The meeting allowed participants to discuss the various health, ecological, economic and social problems generated by the accident as well as their long-lasting character. They underlined the particular importance of re-establishing the affected communities with decent living conditions and developing adapted solutions to the problem of agricultural rehabilitation considering the specificities of the affected local productions. They also emphasized the need to find sustainable solutions for managing the waste resulting from the decontamination program launched by the national authorities that was just starting at that time and to take particular care of health surveillance of the inhabitants.

Building on the Belarusian and Norwegian experiences, which demonstrated the effectiveness of involving the population and the local specialists in managing the radiological situation with the appropriate governmental support, the participants recognized that the Japanese affected population needed further information to understand and evaluate the often-contradictory messages on the consequences of the accident. This information was considered by the participants as crucial to take informed decisions concerning their future in particular to decide whether to stay or leave in their homeland and to return or not.

In a final declaration adopted formally, the participants expressed their willingness to continue the dialogue and called upon the Japanese authorities and international organizations to pursue co-operation on the improvement of living conditions in the affected areas, particularly in relation to engaging local and national stakeholders.

The day after the dialogue, the ICRP representatives met the Japanese minister of environment and the government's working group on the Fukushima accident and presented the final declaration of the dialogue meeting, the ICRP Recommendations on post-accident situations and the lessons learned from Chernobyl in the context of the Ethos project and the CORE programme. A few weeks later, the creation of the "Ethos in Fukushima" group of citizens leaded by R. Ando was announced using extensively social media.

At the same period, the ICRP participants to the first dialogue meeting decided to organize a series of meetings on the rehabilitation of living conditions in the Prefecture, i.e., the so-called "ICRP Dialogue Initiative in Fukushima" (Lochard, 2016). They also decided to invite the "Ethos in Fukushima" members to join these future dialogue meetings.

\subsection{The series of ICRP Dialogue Initiative meetings}

In total, 11 other meetings were organized during the 12 15 period. They were led and coordinated by ICRP with a set of local, national, foreign, and international partner organisations and they mobilized various stakeholders mainly living in slightly contaminated areas but also mandatory or voluntary evacuees and outsiders from other Japanese regions. These meetings were mainly driven by the expectation of the participants to better understand the radiological consequences of the accident, to discuss the main challenges of the post-accident situation faced by the affected inhabitants and for that, to benefit from the experience of experts and communities affected by the Chernobyl accident in Europe. 
Table 1. The 12 meetings of the ICRP Dialogue Initiative (20112015).

1. Rehabilitation of living conditions, 26-27 November 11,

Fukushima City

2. Situation in Date City, 25-26 February 12, Date City

3. Improving the quality of food products, 7-8 July 12 , Date City

4. Education of children and youth, 10-11 November 12, Date

City

5. To return or not, to stay or leave, 2-3 March 13, Date City

6. Facing the situation of Iitate people together, 6-7 July 13,

Fukushima City

7. Self-help actions in Iwaki and Hamadori, 30 November-1

December 13, Iwaki City

8. Situation and challenges of Minami-soma, 10-11 May 14, Minami-soma City

9. Raising children in Fukushima, 30-31 August 14, Date City

10. Value of tradition and culture in Fukushima, 6-7 December

14, Date City

11. Role of measurements in regaining control, 30-31 May 15, Fukushima City

12. Experience we have gained together, 12-13 September 15, Date City

Table 1 presents the topics and places of the meetings of the ICRP Dialogue Initiative. Figure 2 presents the locations of these dialogues on the map of contamination prevailing in May 11. Seven meetings were hosted by the city of Date which was strongly committed in the recovery process.

The two days meetings format was the following:

- discussions with simultaneous translation between Japanese and English;

- thematic sessions with presentations and panel discussions to give the opportunity to all invited participants to express their views but also to favour their mutual listening;

- a final discussion generally followed by the personal comments of T. Lazo of NEA/OECD to close the meeting.

Besides Japanese participants, Belarusian and Norwegian stakeholders (about twenty in total) were invited to share their experience and express their concerns and expectations. Observers (up to several tens) as well as representatives of local media were attending all meetings. The ICRP vice-chair J. Lochard chaired the meetings and T. Schneider and J.F. Lecomte ensured the role of rapporteurs.

Presentations and conclusions of all dialogue meetings were posted on the ICRP web site and the Ethos in Fukushima blog in order to ensure a wider dissemination. The first nine dialogue meetings were financially supported by a group of foreign organizations (IRSN, NRPA, ASN) which all had been actively involved in several projects in the affected areas of Belarus by the Chernobyl accident in the 1990s and the 2000s as well as by NEA/OECD. The Nippon Foundation financially supported the 10th and 11th dialogue meetings. During the 12th dialogue, the ICRP Chair C. Cousins expressed the Commission's support to the affected population and thanked the Japanese stakeholders for their contribution to a better

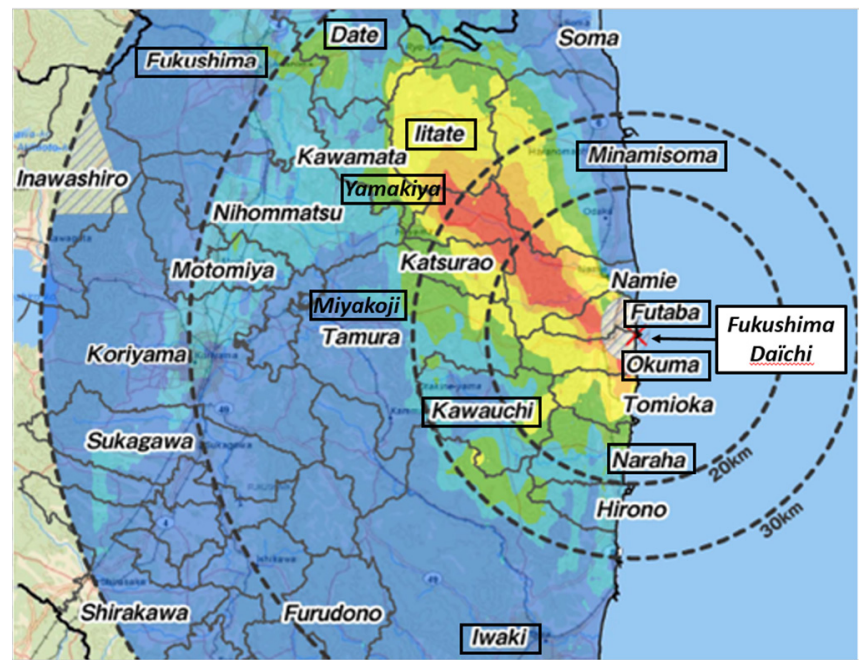

Fig. 2. Locations of the meetings of the ICRP Dialogue Initiative and the Fukushima Dialogues. Ground radiation levels as of May 2011. Source: U.S. Department of Energy.

understanding of the post-accident situation of Fukushima in view of future recommendations of the ICRP.

\subsubsection{Dialogue meetings 2 to 5 in Date City}

Dialogue meetings 2 to 5 took place in Date City located 50-60 km North-West from the damaged power plant and affected by a large range of contamination impacting particularly the local farmers. Rapidly after the accident, the municipality took the initiative to decontaminate the most affected part of the city together with voluntary citizens and NPOs.

Dialogue 2 meeting (Feb 12) aimed at better understanding and discussing the current challenges, the actions implemented so far in the city to improve the radiological situation and the possible ways to improve further the living conditions of the inhabitants. Participants discussed particularly the decontamination programme implemented by the municipality with the support of the Japanese authorities, and the actions taken to help communities to build capacity to take effective self-help protective actions. The local farmers expressed a strong willingness to maintain their activities and culture, with a clear willingness of producing products that could be accepted all over the country. While recognizing the progress made in understanding the problems and the answers provided, they also voiced serious concerns regarding the situation.

Dialogue 3 meeting (July 12) was devoted to the difficulties encountered by local farmers to maintain their activities (mostly large orchards and rice paddies) despite the presence of the contamination. Participants emphasized the need to implement a detailed monitoring to facilitate the identification of clean products and areas needing active management. They also acknowledged the importance to benefit from lessons already learned in Belarus and Norway, and to foster research and experimentation relevant to local conditions for improving the quality of agricultural products. All concerned parties - producers, wholesalers, retailers, and 
consumers, some of them invited by the organizers and coming from uncontaminated areas of Japan - had a constructive dialogue on strategies and management procedures to improve the quality of products and to regain confidence of consumers. Unexpectedly, the participants discussed with great emotion the issue of discrimination of people in the affected areas, especially for those of pre-marital age to marry and have children. They emphasized that this painful issue will not be resolved without the understanding and involvement of people outside the affected areas.

Dialogue 4 meeting (Nov 12) focused on the living conditions of children including rehabilitation initiatives in schools, playgrounds, and their usual living places in Date and the surrounding municipalities. The participants stressed the importance of disseminating a practical radiological protection culture among the children and beyond among adults, based on ethical and cultural considerations in addition to science to empower the affected community. Radiation monitoring was recognized as an important tool to allow everyone to grasp the radiological situation as well as the importance to maintain the memory and experience of the accident among the current generation to ensure their transmission in the future.

Dialogue 5 meeting (March 13) dealt with the very sensitive, personal and emotional issue of "returning or not, staying or not in the affected areas".

Presentations covered the situation in Date city and in other affected municipalities of the Fukushima Prefecture where people were allowed to reside two years after the accident. They addressed the level of individual exposures to radiation, the state of medical care, education and economic activities as well as the current status of the evacuees living within the Prefecture and beyond. They also described the various actions taken by the authorities and by individuals and local communities to rehabilitate the living conditions. The experience of the Sami people in Norway following the Chernobyl accident was also presented, emphasizing the numerous challenges faced by a community living in a contaminated area.

Participants discussed in details the reasons to stay or to leave in the affected areas and the conditions and means to ensure that people can make an informed choice:

- availability of relevant and accessible information on the radiological situation of the areas particularly the effectiveness of decontamination;

- issues related to the human dimension of the situation, i.e., age of the people, quality of life of children and elderly, access to medical services and public health, availability of sustainable infrastructure of the communities, economic activities, creation of new jobs, diversity of shops, effective transports, possibility of preserving family and community ties in the future, socio-economic development of the affected areas, with different challenges according to the communities.

Participants indicated that uncertainties about the future were perceived as increasing rather than decreasing reflecting the growing complexity of the situation: the number of "undecided" about willingness to return raised to about $50 \%$ at the time of the dialogue. They recognized a huge burden on the shoulders of the inhabitants to make their own decision and acknowledged the fact that for some people, because of economic constraints, there is no other alternative than to come back. Ultimately, they stressed that the decisions are those of individuals and it is a fundamental ethical principle that all decisions should be respected.

Nevertheless, it was also mentioned that such decisions are not only an individual choice but may be informed through dialogue at the community level.

In conclusion of the meeting, the participants strongly recommended that:

- national and local authorities should support and participate more actively in dialogue meetings concerning the current challenges and the future of the communities;

- experts, from all relevant disciplines, including psychologists, lawyers, etc., should work together with the local communities to improve their living conditions;

- emergence of local facilitators, particularly among medical staffs, teachers and parents-teachers associations in schools to diffuse practical radiological protection culture should be favoured;

- the objective and effectiveness of the decontamination programme should be revisited with all relevant stakeholders, including the concerned population;

- the evacuees in their new locations should be supported considering the diversity of their situations;

- local initiatives aiming at improving living conditions should be supported and infrastructures to favour sustainable social and economic activities in the affected areas should be developed;

- mechanisms to exchange experience on local initiatives and to diffuse good practices within affected areas and beyond, including abroad, should be established.

It is to note that during the first five meetings, participants emphasized regularly the human dimension of the situation, the particular importance of preserving the dignity of the population and of reinforcing the local, national and international solidarity. They emphasised the importance to develop places of dialogue in the affected communities and to strengthen health surveillance and counselling to deal with the trauma and distress caused by the consequences of the accident.

They also stressed that inhabitants are the owners of the situation in the affected areas and in order to better address their expectations experts should be at their service, listen to their concerns and respond to their questions in an understandable way. The implementation of joint evaluations of the situation by evacuees, residents, experts and authorities in the various communities (the so-called co-expertise process) was emphasized as well as the importance to develop practical radiological protection culture among the population and notably at school. Participants recognized the need for a more detailed characterisation of the radiological situation to help individuals to know where, when and how they are exposed and what can be done to improve their own situation. Many of them expressed their expectation to enjoy the daily life again in their communities as well as their hope for the future. They mentioned the shared ethical duty with regard to the history and the future of their lands and communities and they emphasized the importance to preserve natural landscapes and to transmit local culture and traditions. 


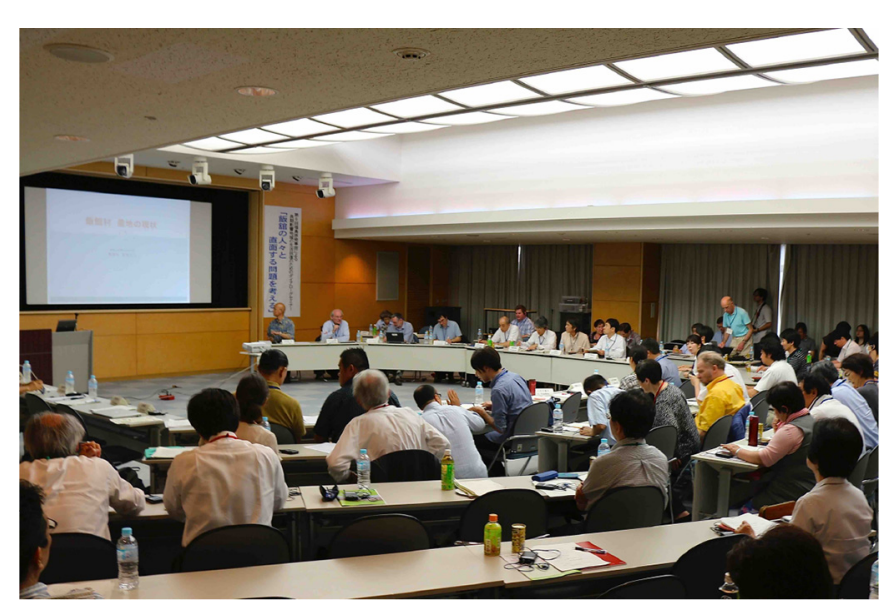

Fig. 3. Dialogue meeting 6: Facing the situation of Iitate people together, 6-7 July 2013, Fukushima City.

\subsubsection{Addressing the challenges of litate, Iwaki and Minami-soma}

Dialogue 6 meeting (July 13) discussed the present situation and challenges of the citizens of Iitate located North West of the emergency evacuation zone (Figs. 2 and 3). On the basis of ambient radioactivity measurements, the authorities decided to evacuate Iitate on the 22nd April 11. People were then cut off from their own land and lived in exile. At time of the dialogue more than two years later, they were facing the difficult decision of whether to prepare for returning to their home or not. Some had already chosen to live in a new place and not to return to Iitate. Others were still grappling with the complexity of the situation, hesitant in their decision to return or not, and feeling the pressure of passing time. The objective of the dialogue was for a range of stakeholders, organizations, inhabitants, teachers, doctors, authorities and foreign participants to share the situation of the Iitate people, to understand their problems, and to discuss the way forward.

Presentations were made on the radiological situation of the village, on difficulties and challenges the people of Iitate were facing and actions already undertaken to improve their living conditions.

During the panels, Iitate people expressed anger, emotions, doubts and mistrust. They explained that they had lost so many things and never received personal apologies from TEPCO or the government. Many promises had not been kept and it was difficult to have hope for the future as long as the past has not been solved. The information provided by authorities and experts was difficult to understand and equally difficult to use directly by the inhabitants to cope with the current local radiological situation. Iitate villagers recognized a lack of individual knowledge about the radiological situation needed to allow them to move forward. After the accident, their voices had not been listened to and they had no places to express their views. In addition, living in exile has weakened the bonds between people in Iitate, making sharing difficult and enhanced the feeling of isolation, leading to difficulties in reaching consensus within the community on what to do. They emphasized that, although the decontamination programme was viewed by the villagers as a significant step to improve the radiological situation, they considered its implementation to have been too slow, and lacking a clear strategy, timetable, and leadership. They also recognized that it was impossible to completely remove the contamination.

Finally, four main recommendations emerged from Iitate villagers:

- create places of dialogue respecting the expression of different points of view, favouring the exchange of information and allowing villagers to define their own projects;

- provide equipment and devices to allow villagers to make their own measurements and establish a framework of cooperation between them, researchers and experts to develop projects at the service of the population;

- define priorities for decontamination actions in Iitate and to consider all other actions to reduce the exposure of the villagers;

- create as soon as possible the conditions for elderly people to make their own decisions concerning on whether or not to return to Iitate.

Dialogue 7 meeting (Nov 13). The city of Iwaki received after the accident a large number of evacuees from the heavily contaminated region of Hamadori.

The presentations and discussions focused on self-help actions developed in Iwaki and outside communities by local people to improve their living conditions. This dialogue was the first one to have a series of testimonies about how people and communities mobilized themselves with the support of experts to understand the situation in their immediate surroundings and to implement actions to master this situation. Presentations and discussions showed that recommendations from the previous dialogues, such as the importance of individual monitoring, self-help protection, exchange of experience in places of dialogue, and the role of expertise serving the needs of people and communities, could be implemented in practice and were also very effective in helping to rebuild a decent life and reclaiming their familiar environment.

Dialogue 8 meeting (May 14). Minami-soma underwent the triple disaster of the earthquake, the tsunami and the nuclear accident, suffered from a partial evacuation order without allowing residents to search for missing persons and was still facing an extremely complex situation in May 14 .

The city had been divided into three areas: the evacuation zone, the in-house sheltering zone and other areas with special spot recommended for evacuation. Altogether, $90 \%$ of the people were evacuated. At the time of the dialogue, only $50 \%$ were back and they were struggling to rehabilitate the living conditions in the affected part of the city with the help of experts.

The dialogue focused on the situation and challenges of the local people but also gave the opportunity to residents of the communities of Suetsugi, Kanagawa, and from the highland of Norway affected by the fallout of the Chernobyl accident to share their experiences. The participants discussed how to overcome the fragmentation of the community due to the division of the city into different zones and its isolation from the rest of the country due to the interruption of the main accesses by train and road to the city. They finally shared visions on how to reconstruct the future of their community. 


\subsubsection{Preparing the future}

Dialogue 9 meeting (August 14) was devoted to all questions related to raising children in Fukushima Prefecture after the nuclear accident. The meeting gathered current and future parents, educators, physicians and professionals dealing with early childhood and concerned about the future of children of Fukushima Prefecture.

Presentations by fathers and mothers revealed that family members were often divided regarding how to deal with the protection of children, particularly young ones. Several medical professionals showed that overprotection of children from the potential effects of contamination, notably by restricting their outdoor activities, had led to indirect effects detrimental to their health and social life. How to protect children while ensuring their psychomotor development and the question of whether or not to develop specific advice for them were debated with passion during the panels. Participants recognized it was a complex issue that appeals to many ethical values and there were no ready-made solutions. Participants recognized that families were facing difficult dilemmas and their choices should be respected. Ultimately, the attitude to be adopted was depending on the particular circumstances, including the radiological situation, in which the children were raised.

The discussions also stressed that it was important to provide a forum for children, particularly teenagers, to allow them to express their feelings, exchange experiences and also speak about the problems concerning life in a contaminated territory. This raised among the participants the question of the role of parents and educators in the dissemination and transmission of a practical radiological protection culture among young people to protect themselves and take informed decisions in a contaminated environment, which goes well beyond the acquisition of scientific bases about radiation at school.

Dialogue 10 meeting (Dec 14) had for theme "The value of tradition and culture in Fukushima". In addition to presentations on the daily cultural life and festival tradition in Fukushima Prefecture, this meeting also included traditional drumming and dancing performances (Fig. 4). Moreover, a group of villagers from Iitate who had recently visited the areas affected by the Chernobyl accident in Norway presented their travel impressions. At the occasion of this visit, they met again the Norwegian farmers who participated to the Minami-soma dialogue the former year. This was for the Iitate villagers the opportunity to be directly confronted to the daily life of persons whose tradition and culture had been profoundly threatened at the time of the Chernobyl accident (Liland and Skuterud, 2013). The panel discussions highlighted the fact that the lack of meaningful information and knowledge on how to behave in front of the contamination that prevailed after the accident, combined with conflicting information on the effects of radiation on health led to a great confusion among the population. This resulted in the loss of shared values among people in the affected areas, leading to the fragmentation of families and communities.

However, despite this profound disturbance of family and social ties, the ancestral values underpinning the cultural life of Fukushima had been preserved and had contributed to maintain a link between those who stayed as those who left

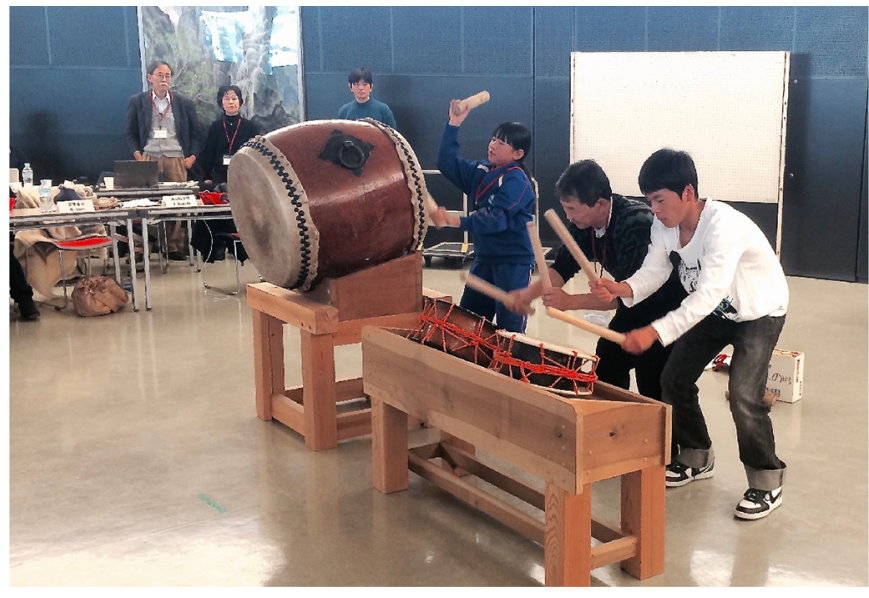

Fig. 4. The traditional drumming performance at the 10th dialogue meeting in Date City in December 2014.

the Prefecture. Tradition and culture are in all dimensions of Fukushima people's life, from daily life activities of eating and working, to the perpetuation of local festivals, which the community preserved for centuries. Sharing the values that constitute this cultural heritage was considered important because it was a bulwark against the creeping disintegration of society caused by the presence of radioactivity. It was seen at the same time as the source of inspiration and creativity for building the future of Fukushima. The tenth dialogue meeting demonstrated that the local tradition and culture were still strongly alive in the communities and were functioning to keep the solidarity of people in the affected areas of the Prefecture.

Dialogue 11 meeting (May 15) addressed "The role of measurements in regaining control". A series of presentations made by inhabitants and representatives of citizen groups or organizations involved in measurements in the contaminated areas after the accident were delivered. These presentations, and related discussions, highlighted the importance of measurements to help individuals, families and communities to progressively understand the radiological situation and take informed decisions about their daily life. The results presented included all measurement categories allowing people to know the radiological situation of their own surroundings and thus to orient themselves in their daily life: ambient dose rates, food contamination measurements, individual external dose and whole-body contamination. Participants emphasized that through measurements, people not only were regaining confidence but also by sharing results with anyone living in the same areas, bringing the tie between people back again and restoring solidarity.

In conclusion, although participants recognized that most results concerning individual exposures were lower than expected, they pointed out the importance of continuing the radiation monitoring and of organising a long-term vigilance to identify specific groups with higher exposures.

Dialogue 12 meeting (Sept 15) dealt with "Experience we have gained together'. In the presence of the ICRP Chair, C. Cousins, its objective was to review the path and steps of people in and out of Fukushima Prefecture, during the four and a half years after the disaster. Participants to previous dialogue meetings were invited to share their views and experience about past, present, and future lives. The dialogue allowed to 
report about the Fukushima situation by the end of summer 15 from a multiple perspective by inviting not only people from Fukushima but also those from outside of the Prefecture who engaged with the affected population to rehabilitate the living conditions. It was stressed that, although many protective actions had been implemented and the socio-economic conditions had been partly resettled in several municipalities, there were still numerous problems to be solved. Some people were moving forward, but some were feeling isolated or still had to cope with situations more complex than before 11 and significantly deteriorated living conditions. At the end of the meeting, participants considered that managing the Fukushima situation was less a scientific issue than a matter of stakeholders working together.

Since the accident in the contaminated areas, mothers learned how to take care of their children, farmers how to promote good quality products, teachers how to develop practical radiological protection culture, journalists how to disseminate relevant information on daily life, experts how to be at the service of the population, and authorities how to provide information and support, and respect individual choices. Box 1 presents some words of participants expressed during the panels.

\section{Box 1. Words of participants to the 12th dialogue meeting.}

"Each of us has its own life beyond the radiation issues."

"Numbers should not control our lives; they should help us make our lives better."

"The hope is to see a bright side for the future."

"We have to respect each route."

"It is my role to prepare the village for the future, for my children."

"Fukushima people lost something forever. It is not possible to forget. But at the same time, we have to move forward."

\subsection{The International Workshop on the ICRP Dialogue Initiative}

Decided at the occasion of Dialogue 6 meeting in summer 13, the International Workshop that closed the ICRP Dialogue Initiative took place in December 15 with the objective to share the experience and main lessons of the twelve meetings. Hosted by Date City the event was organized by ICRP in cooperation with the municipality, Ethos in Fukushima and a series of Japanese organisations: FMU, Fukushima Prefecture, Japan Health Physics Society, Japanese Cabinet Office, the Japanese Nuclear Regulatory Authority, Ministry of the Environment of Japan, Radiation Safety Forum Japan and the Nippon Foundation. It was supported financially by IRSN and ASN (France), NRPA (Norway), and NEA-OECD.

Under the title "Rehabilitation of living conditions after the nuclear accident" the programme of the workshop included a first session dedicated to an overview of the situation in the Fukushima Prefecture almost five years after the accident, a second session presenting personal testimonies of participants to the dialogue meetings, a third session presenting the lessons learned by the participating organizations from abroad, and a fourth one presenting the views of the participants to the dialogue meetings about the future of the Prefecture.

Box 2 presents some of the words reflecting the key messages delivered by the Fukushima participants (ICRP, 2016).

\section{Box 2. Words of the International workshop on the Fukushima Dialogue Initiative.}

"The Great East Japan Earthquake of 11 March 11, the tsunami, and living in displacement following the nuclear disaster have taught me that: (1) anything can happen in life; (2) measurement and data are important to have an accurate grasp of the situation; (3) networking with people through the ICRP Dialogue seminars enabled me to take measurements; and (4) my family spurred me to action. It was important to have an accurate grasp of the situation so that my family could live in peace, and taking measurements was necessary to understand the situation. These measurements also provided hope. The data became a 'shield' from inaccurate information and false rumours, and protected my family and I from unnecessary anxiety." K. Kanno

"In Fukushima, what we need in the future is ... to be with and listen to people who need to be heard. Education and human development are very important, and experts who can speak the same language, with simple and comprehensible words, are indispensable." M. Otsuki

"Another important lesson was the establishment of an environment for residents to come together to talk. It is this form of communication that allows differences of opinion and feelings to be discussed openly, so no views are ignored and discarded. There have been many valuable encounters with people we would never have met had it not been for the ICRP Dialogue seminars. This is indeed one of the great achievements of the seminars, without which we would never have been able to listen to diverse opinions and experiences from so many people." T. Hanzawa

"In the wake of the accident, we, in Fukushima Prefecture, have continued our efforts to communicate amongst ourselves. The results of our endeavours are nothing glorious or conspicuous, but I feel that they have definitely contributed to the improvement of people's living environments. I would like to conclude... with a recommendation that the efforts of dialoguing are not only for the people of affected areas, but also for relevant groups such as experts and administrators." R. Ando

For the international experts, the testimonies during the workshop confirmed that the sudden presence of radioactivity has profoundly disrupted people's lives and the lack of basic knowledge about radiation and radiation protection combined with the unpreparedness of such a situation has increased their distress and difficulties. Several experts emphasized that 
nuclear emergency preparedness planning should not be limited to evacuation and iodine prophylaxis, but should include plans for the subsequent phase of recovery and the implementation of long-term protective measures.

Furthermore, the testimonies also confirmed that radiological protection following a nuclear accident should be focused on the rehabilitation of living conditions and on the restoration of the dignity and well-being of individuals and the quality of the living together. The ICRP Dialogue meetings did not raise new issues concerning the protection of people living in longterm contaminated areas, calling for a significant change to the principles, criteria, and advice of ICRP. However, they brought several important clarifications and complements concerning the human and organisational dimensions of the rehabilitation process, and the challenge is now to incorporate these elements into recommendations and guidelines in preparing for the management of possible future accidents. The proceedings of the workshop have been published by ICRP with the support of the Nippon Foundation and the other financial supporters (ICRP, 2016).

Beyond the series of presentations, the hundred or so participants, among which many participants to the dialogue meetings and the General Directors of NEA, IRSN, ASN and NRPA, had also the opportunity to discover the Web documentary "Kotoba" that tells the story of the four years of dialogue meetings. The documentary uses the dialogue as a thread, but the stories are primarily those of people for whom the accident and recovery have been an ever-present and enormous part of their lives. It was commissioned by IRSN with the support of NRPA (Kotoba, 2015).

\section{3 "The Fukushima Dialogue" meetings (2016-2018)}

A second series of eight meetings named "The Fukushima Dialogue" marked a significant evolution of the organization and objectives of the dialogues.

Attended by residents of communities for whom the evacuation order had been or was about to be lifted, their purpose was mainly to share the problems these communities would have to face in the medium term to restore decent living conditions of the residents. They were also an opportunity for many national and international experts and students from Japanese Universities to better understand the issues faced by these communities and for Fukushima participants to develop and strengthen a network of citizens and local and national experts in the Prefecture.

The two days meetings format was on average the following:

- the first day was dedicated to visit the host community and to meet with local residents, and;

- the second day had presentations in a meeting room in the morning and a panel discussion in the afternoon. The chairmanship and the facilitating of the meetings were assured by J. Lochard and R. Ando.

All meetings of "The Fukushima Dialogue" were entirely supported financially by the Nippon Foundation. ICRP kept a consultative role and remained in charge of organizing some
Table 2. The 8 meetings of the Fukushima Dialogue (2016-2018).

1. Situation of Miyakoji today, 12-13 March 16, Miyakoji village

2. Sharing experiences in Iitate village today, 9-10 July 16 , Iitate village

3. Rehabilitation of living conditions in Futaba region, 1-2 October 16, Kawauchi village

4. Current situations of locals near the intermediate storage facilities and surrounding areas, 11-12 March 17, Futaba and Ohkuma towns

5. What do we need for our future?, 8-9 July 17, Date City

6. Dialogue with residents of Yamakiya, 25-26 November 17, Yamakiya village

7. Current situations and future of Odaka area, 10-11 February 18, Minami-soma City

8. After Fukushima Nuclear accident: preserve memory, share experience and go toward the future, 15-16 December 18 , Iwaki City

local logistics and the participation of its members and foreign guests.

Table 2 presents the topics of the 8 dialogue meetings held between 16 and 18 with their dates and the Figure 2 presents their places.

The Fukushima Dialogue 1 meeting (March 16) took place in Miyakoji, a village located 20 to $30 \mathrm{~km}$ west of the Fukushima power plant and evacuated at the time of the accident. In the course of 13, the authorities had lifted the evacuation order but the situation remained in 16 very difficult and only a few inhabitants had returned.

The meeting was initiated by O. Niwa, R. Ando and M. Miyazaki (FMU), a frequent participant in the dialogue meetings, who had organized in May 14 a visit with ICRP members to the village to meet a farmer who had resumed his activities and the school director who was waiting for reopening the establishment, in order to better understand the situation in the municipalities in which the evacuation order had just been lifted.

The Fukushima Dialogue 1 meeting was a follow up of the May 14 informal visit and of the international workshop of December 15 .

The group of twenty visitors, including representatives of Japanese authorities, ICRP members and French experts of IRSN, visited the village, met the inhabitants and exchanged with them in the communal house. The group met the director of the kinder garden to discuss her challenges and to evoke the difficulties encountered by the young families who decided to return to Miyakoji. The visit continued with the farmer met in May 14.

A more formal meeting took place in the guesthouse that had recently been reopened to discuss with local authorities on the process that had led to the decision to lift the evacuation order. M. Miyazaki presented the role played by the measurements of individual doses with the dosimeter "D Shuttle" making it possible to link the external dose and the activities of the person (Chiyoda Technol, 2018). After discussing with all participants, it appeared that these measurements contributed to the decision of the villagers to allow the lifting of the evacuation order by the national authorities. 
This is at the occasion of the Miyakoji meeting that the Japanese participants agreed to continue the dialogue meetings and to rename them "The Fukushima Dialogue" to mark the fact that from now on the organization and programmes of the dialogues passed essentially in the hands of the local stakeholders and participants but still in cooperation with ICRP. This was reflected on the Ethos in Fukushima Web Site ${ }^{1}$, which has continued to be used for announcing the programmes and diffusing the material of the meetings.

The Fukushima Dialogue 2 meeting (July 16) took place in litate village three years after the preceding dialogue. Because the evacuation order was still in force, several very determined villagers had taken actions to address and overcome the various problems that litate had been confronted with during this difficult period. In this context, the objective of the meeting was for the villagers, five years after the accident, to discuss on site the current situation of the village and to share their plans for the future. Several participants to the ICRP dialogue 6 meeting in July 13 dedicated to Iitate attended this dialogue together with new participants.

On the first day, the group of around forty people including Japanese and foreign experts visited six places/persons in Iitate:

- a farm in which the owner had undertaken various actions in collaboration with universities and research institutes concerning the measurement of contamination, decontamination experiments and above-ground cultivation;

- another farm in which the farmer was burying the surface soil of ancient rice fields under deep soil layers in order to convert them to pasture;

- a couple who had just expanded their house with logs of cedar trees that were surrounding the house and which had been cut down during the decontamination work. After having measured the contamination of the logs, they had decided to use them to carry out the expansion project which was dear to their heart;

- an experimentation site investigating the effects of the burial of decontamination waste on the quality of water. As the water supply to Iitate is at high altitude where the decontamination sites are located, residents feared that the radioactivity would migrate to the village;

- an elderly nursing home, which was allowed to continue to operate despite the evacuation order in May 11. The director of the establishment explained that having measured the ambient dose rates all around the building, it was decided not to evacuate given the burden this would have placed on patients. He also explained the constraints on the staff forced to make long journeys every day to get to work;

- and finally, a local manager who had been in charge of ambient dose rate measurements before the decontamination. Regarding the problems that will arise after the lifting of the evacuation order, he stressed the difficulty of maintaining and managing the cultivated lands in the next 5 to 10 years in a place where it is impossible to make a living by taking over agriculture. He emphasized that concretely, the most important point for maintaining cultivated land will be to control the creeping weeds in summer

\footnotetext{
${ }^{1}$ Ethos in Fukushima. http://ethos-fukushima.blogspot.jp/p/icrp-dia logue.html and http://ethos-fukushima.blogspot.com.
}

and also to investigate the possibility of introducing alternative crops such as buckwheat.

During the second day, several relatively old people expressed their views about the situation and the way they see the future of the village. The elderly people expressed a rather positive view concerning the lifting of the evacuation order and the rehabilitation actions implemented in the area. As for young people, some of whom had already established a new life In Fukushima City, they said they could hardly imagine the immediate return to which they were invited. Such a return should be contemplated in a longer time span of 10 to 20 years for their own life plan, or for their village and families.

Fukushima Dialogue 3 meeting (Oct 16) in Kawauchi, organised in cooperation with Nagasaki University and IRSN, focused on the rehabilitation of living conditions in Futaba region. The evacuation order was lifted in 11 for Hirono, in 12 for a large part of Kawauchi and more recently for the rest of Kawauchi, Naraha and Katsurao, highlighting the question of returning home. At the time of the dialogue meeting, some inhabitants had already decided whether to return or not, while others were still searching for the best decision for themselves and their relatives.

Participants included residents, authorities, experts, and NGOs who shared experiences and addressed the challenges related to the rehabilitation with a focus on the implications of decontamination, in particular the issue of long-term waste management.

The first part of the dialogue was based on testimonies of inhabitants from four villages, round tables and exchanges between all the participants:

- the mayor of Kawauchi explained that at the time of the accident, the village located $20 \mathrm{~km}$ away from the coast had welcomed a large number of evacuees from Tomioka and on the 16th of March 11, all the village was evacuated to Koriyama. Due to the low contamination in a large part of the village, the evacuation order was lifted at the beginning of 12. The mayor clearly emphasized that the key words for the return to Kawauchi were: respect of choice of each resident, self-support to encourage the residents to restart their life and trust in order to build a good relationship between residents and local authorities;

- the representative of Hirono explained that the evacuation was limited to few months and that the basic principle for the municipality was to provide safe living conditions and environment for their inhabitants;

- for Naraha, the representative of the municipality mentioned the specific situation with $80 \%$ of the town within the $20 \mathrm{~km}$ zone. Due to the long duration of evacuation period, it was noticed at the time of the dialogue meeting the decreasing willingness of the inhabitants to return home;

- the representative of Katsurao presented the situation after the lifting of evacuation order in June 16. Katsurao being a small village, the major issue was to restart agriculture activities.

The second part of the dialogue was dedicated to social and economic prospects of returning as well as to waste management and environmental issues. Participants expressed their view on the current situation. 
The lack of medical services was emphasized with particular concern for elderly people, children and pregnant women. Many people were still living in temporary houses notably because of the difficulties to restore or rebuild the damaged houses. Among the issues to be addressed for considering the return to homeland, the lack of economic development and the difficulties encountered in the restart of the agricultural sector were clearly quoted together with the evolution of the local communities following the accident, including the workers involved in the decommissioning activities. However, they also mentioned the crucial role of the festivals and revival of local cultural events for the communities to be again together.

For waste management and environment, the key concern expressed by the participants was the contamination of forests and mountains with doubts about the efficiency of the decontamination process for the environment, its cost as well as the amount of waste produced. They also mentioned the risk of transfer with rain of radionuclides present in the forest. Finally, they called for a better access to information notably related to the contamination of the environment, the level of exposure for each individual, the control of waste incinerators located in the village, the efficiency of decontamination and the quality of local agricultural production. It was clearly expressed that the management of waste from decontamination activities is not only the problem of people from Fukushima Prefecture but of Japan and even worldwide and participants wished an increase of solidarity.

Finally, the role of experts to help understanding the information was emphasized, although the participants mentioned that experts have sometimes controversial opinions!

Fukushima Dialogue 4 meeting of Futaba-Ohkuma (11-12 March 17). Mostly located in a "difficult to return zone" the two villages close to the Fukushima Daiichi nuclear power plant still had restricted entries and remained confronted with a very difficult situation not well-known by the general public even if there was some recovery, particularly in terms of infrastructures, in areas where people lived or were soon able to live.

The field visits with mandatory protective clothing (Fig. 5) started at a resident's house located $1.5 \mathrm{~km}$ northwest from the nuclear power plant. After telling how he had lived through the accident and the events that followed, the owner invited the participants to visit his house totally devastated not only by the earthquake but also largely after the evacuation by wild animals, especially wild boars, that had broken doors looking for food. He explained that having lost everything built up in his life, he became depressed and sometimes imagined committing suicide but he regained the will to live by seeing grow his grand-son, born at the time of the accident.

Then participants visited a temple where festivals were held before the accident. Some villagers visited the graves of the cemetery on the opposite side of the temple to honour the dead on this 6th anniversary day of the earthquake. Then participants visited a community centre where cultural activities used to take place in the past. Drums used for these activities were abandoned without being touched.

On the second day, the dialogue took place in a newly rebuilt nursery school in Nahara with presentations on the general situation of Futaba, on the results of dose measurements in the houses of Ohkuma and Futaba, on the project of

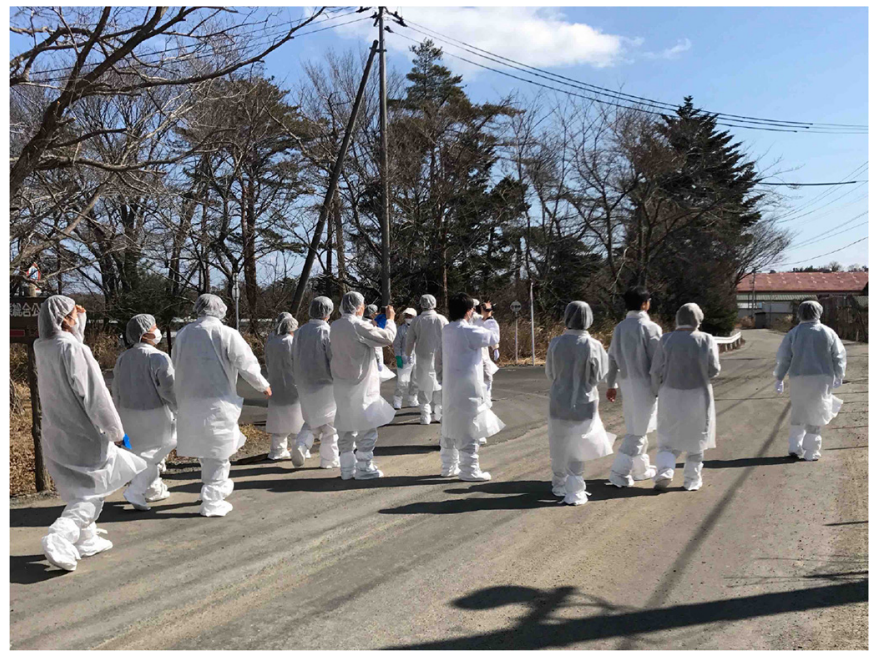

Fig. 5. The field visit during the Fubata-Ohkuma dialogue meeting in March 2017.

implementation of the intermediate storage facilities (ISF) for the waste of the decontamination from all over the Prefecture and on the reconstruction plans for the future. There was also a presentation about a traditional dance of Futaba followed by a description of the festival activities of the area in the past.

Local participants to the final panel narrated how they became depressed for a few years after the disaster, disappointed on what to do and with temptation to commit suicide. They also explained how each one discovered ways to move forward, e.g., by actively planning gatherings in places for refugees. They often expressed words of love for their hometown and explained how they tried to remain positive in their new living places, still keeping strong sympathy for their hometown.

The dialogue meeting was an opportunity for locals to be heard, to express their concerns, and to provide a platform for further discussion on the type of future they want. In the closing session C. Cousins, ICRP chair who was participating for the second time in a dialogue, stressed how she had been deeply touched during the field visit and that it was important for radiological protection professionals to figure out the human dimension of a nuclear accident. She reiterated the importance for the ICRP of being part of the dialogue meetings.

Fukushima Dialogue 5 meeting (July 17) in Date City addressed the question "What do we need for our future?". It was the first time to meet again in this place in about one and a half year after the International Workshop on the Fukushima Dialogue Initiative.

Following the lifting of the evacuation order two months before in April everywhere except in the difficult-to-return zones Fukushima people had recovered their freedom of residence but the social and psychological effects due to the long evacuation orders were still very pregnant. For the difficult-to-return zones, where the status of the evacuation order was still unclear, the situation was even more difficult. Under these circumstances, it became difficult for them to grasp the complexity of the present situation, and even more difficult to share a common vision of the future of the places in which they had chosen to live. The dialogue meeting aimed to 
exchange views between people who lived in the Fukushima Prefecture and had attended the previous dialogue meetings and also to express their expectations and hopes regarding the recovery process.

Presentations during the dialogue meeting addressed:

- the situation in several communities of the Prefecture;

- problems associated with the recovery of agricultural activities after the evacuation orders were lifted and the role of the Fukushima cooperative to support producers and consumers;

- actions of the nursery school to accompany the recovery process.

A special testimony was given by A. Fiadosenko from a Belarusian village located in the $30 \mathrm{~km}$ forbidden zone of Chernobyl. She reported about her experience of evacuating the village with her family 10 days after the accident, participating as "liquidator" to the evacuation of life stock from the $30 \mathrm{~km}$ zone, becoming a dosimetrist in a collective farm village adjacent to the forbidden zone where she lived until now, participating to the CORE Programme to measure the contamination of foodstuffs of private production and working with the local population to improve the protection of young children against internal contamination and finally her present role of transmitting her experience to the next generation.

The local participants evoked the complexity of the rehabilitation process, the diversity of situations in the different places where they lived. Because the actions held in various places in the Prefecture were seen as isolated, efforts had to be deployed to connect people and share experience by transmitting information in the Fukushima Prefecture for the success of the recovery process. Participants discussed the role that the dialogue meetings should play in the rehabilitation and the organisers of the Fukushima dialogue envisaged the creation of a newsletter to share the experience gathered in the dialogue meetings.

IRSN President D. Le Guludec and Director-General J.C. Niel participated in this dialogue meeting.

Fukushima Dialogue 6 meeting (Nov 17) with residents in Yamakiya. Six years after the accident, the evacuation order was lifted on the 31st March 17. However, many remaining challenges were discussed: life infrastructure, agricultural production, temporary storage facility, aging of population and the limited contacts of the returning residents with those living in the same district.

Several visits took place during this Dialogue meeting:

- visit to the farmers of the highlands of Yamakiya district where agriculture and cattle and horse grazing had been major historical economic activities;

- visit to the Yasaka Shrine where the traditional dance annual ceremony (Sanbiki-shishimai-dance of three lions) with a history of more than 300 years was stopped by the evacuation order. It resumed in October 17 with adult performers replacing the traditional young boys around ten years old because there were few children in the district;

- visit to the farm of K. Hirono who cultivated lisianthus flowers. The production stopped following the evacuation order. She was allowed to resume her production before the lifting of the evacuation order and was encouraged to do so by her family and retailers. Her biggest trouble was the lack of manpower and she was ready to hire young people;
- visit to the forest verification site where Chiba University experts continuously observed that ${ }^{137} \mathrm{Cs}$ stays in black and soft soil of the forest within about five $\mathrm{cm}$ depth, and with no tendency to sink into the deeper soil, and absorption of ${ }^{137} \mathrm{Cs}$ into timber varies with tree species;

- visit to one of the 22 temporary storage sites of decontamination wastes located in Yamakiya district;

- visit to the greenhouse cultivation of the mini tomato farm of K. Ouchi ruined by the accident and evacuation during which he visited his house twice a day to feed his dog. K. Ouchi was preparing to start greenhouse cultivation of anthurium, a product without concern for reputational damage due to the contamination, but with no expectations for official supports for restarting his activity.

Farmers, local authorities, representative of agriculture association as well as university experts explained the difficulties they were facing in order to reorganise and restart the agriculture with a limited number of farmers, imposing to come back to traditional family activities and a new management notably for the irrigation of the field.

Participants also reported the importance of the recent restoration of roads improving the accessibility to the district, as well as the installation of a new medical clinic and a firemen station. They also emphasized their attachment to the beauty of the landscape of their homeland to be preserved (not utilised fields). They highlighted some remaining challenges such as the lack of manpower, the aging of the population, the necessity to reorganize the irrigation (need to clean all the channels) and the false rumours about contamination of the products coming from Yamakiya. Finally, they acknowledged the support of the agricultural development association and of experts from universities and national institutes, and the emergence of solidarity of people from outside the Fukushima Prefecture, notably students from Hiroshima University.

Fukushima Dialogue 7 meeting (Feb 18) in Minami-soma as a follow up of ICRP Dialogue 8 meeting (May 14). It focused on the challenges and the future after the lifting on July 16 of the evacuation order of the Odaka area and a part of Minami-soma allowing most of the residents to return to their homes.

The field visits started with the area devastated by the tsunami, now transformed into a no-man's land, bordered by a dike. Then participants met the few farmers (mainly over 60 years old) who experimented agricultural productions during the period of evacuation and were ready to start again their activities after the lifting of the evacuation order. A large number of fields were now covered by solar panels or dedicated to waste storage.

A series of testimonies presented the efforts made for the decontamination of the affected areas and for the collection of debris induced by the tsunami. Participants reported on the slow progress in reconstruction of infrastructures, the reorganisation of school (re-opened in April 17) and the efforts to set up robotic industry in the region.

Inhabitants testified on their living in temporary houses with limited space, their disturbed family life and the feeling of exclusion from hosted communities during the evacuation period, before their return to Odaka where the social structure and the day-to-day activities had been greatly changed after six years of evacuation. They reported their concern about the 
reconstitution of the local community and notably the lack of young people, and about the necessary support in health care to be provided to elderly people.

Farmers presented the challenges faced to restart their activities:

- training to understand the radiological situation;

- securing the fields from wild animals;

- putting products on the market with reluctant consumers and finally mainly used for feeding animals.

Participants also expressed their worries about the degradation of their familiar landscapes. They emphasized the importance for them to keep the memory and the traditions of their community, e.g., the festivals. Finally, they mentioned that this period of recovery pushed them to identify what was crucial for them in order to preserve the potential for a sustainable future for the next generations.

A representative from NRPA presented the challenges faced by the Sami community in Norway following the Chernobyl accident, which were in some aspects similar to the situation of the inhabitants from Minami-soma.

Fukushima Dialogue 8 meeting (Dec 18) in Iwaki. Because the memory of the events gradually fades with the years after the accident and affected people have the fear of being abandoned the issue of this last dialogue meeting was: "After the Fukushima Nuclear accident: preserve memory, share experience and go toward the future".

During the dialogue, a large variety of testimonies, points of view, stories and actions experimented by affected people coming from different Japanese cities/villages and also from Belarus were expressed and shared. Questions addressed during discussions were:

- What to share and with who?

- What should be passed on the next generation?

- How to preserve and transfer the memory?

The participants quoted the interest of sharing emotions, facts (including human and scientific data as well as local, regional or national events) and points of views, focusing on the reality of the large variety of situations, on corresponding experiments and lessons drawn. Several participants confessed that they did not know what the affected people had lived and are currently living.

Significant points to address and possible future actions to strengthen the sharing and transmission of the experience of the accident to the present generation and future generations were identified:

- the fact that the situation evolves over time;

- the necessity of a mutual understanding to avoid misinformation, false rumours as well as to reconnect people, and to rebuild relationship, even inside families themselves;

- the education of the young generation who should be able to talk confidently of the accident;

- the preservation of the memory at both individual and community levels;

- the creation of a museum similar to Braguin's museum in Belarus;

- the importance of ferrymen for preserving and transferring the memory to the different communities and generations.

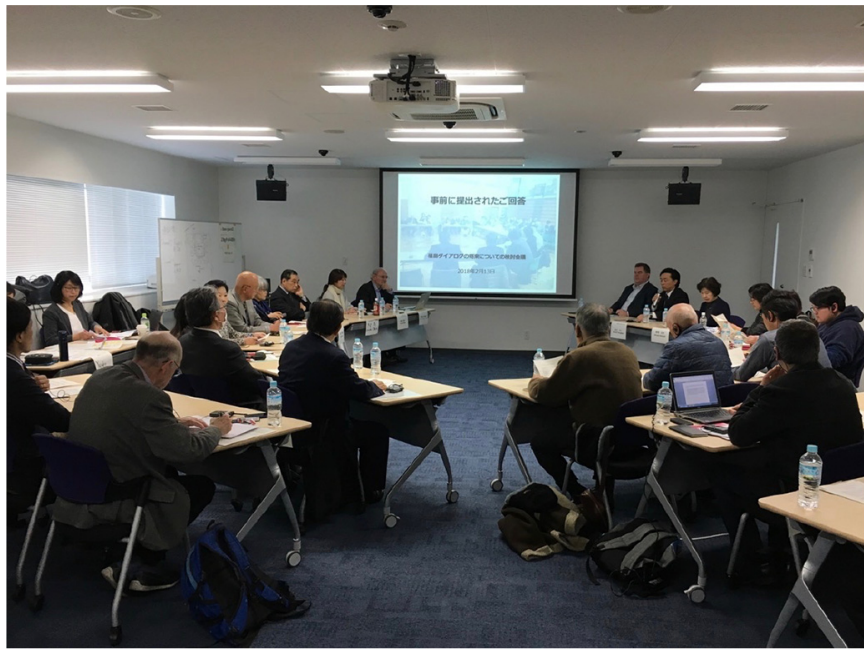

Fig. 6. General view of the strategic meeting at Fukushima Medical University (13 March 2018).

\section{The future of the Dialogue meetings}

\subsection{The strategic meeting on 13th February 2018}

A strategic meeting held on the 13th February 18 at the FMU premises brought together about thirty participants representing the different parties involved in the organization of the dialogues: Ethos in Fukushima (EiF) and other local stakeholders, foreign experts (ICRP, NEA, IRSN, NRPA and CEPN), as well as a representative of the Ministry of the Environment (MoE) (Fig. 6).

The objectives of the strategic meeting were for local stakeholders having actively participated to the Dialogue meetings to:

- draw the lessons of the 19 Dialogue meetings held so far;

- share what influence the Dialogue meetings had on the rehabilitation process in Japan and abroad;

- explore how the main results of the Dialogue meetings could be disseminated;

- express their views concerning the possible continuation of the dialogue;

- suggest the way future meetings should be organized, the topics to be addressed, and the diffusion of their results in case the Dialogue meetings continue.

The main elements that emerged from the discussions were as follows:

- the Dialogue meetings gave the opportunity to participants to meet other people from Fukushima Prefecture but also from the rest of Japan and the world, to share information and experiences, to obtain objective scientific information, and to express freely personal views on the different situations they faced;

- the presence of international experts has been for participants a factor favouring confidence in the information provided on radiation risk and an effective means to alleviate mistrust about scientists, experts and authorities; - the Dialogue meetings allowed participants to be empowered about the scientific issues related to radiologi- 
cal protection and in particular to make the connection between radioactivity and other aspects of the situation resulting from the accident;

- several local initiatives have been inspired by the Dialogue meetings, e.g., the exemplary co-expertise process implemented by the Suetsugi community, and actions involving stakeholders like in Iitate, Kawauchi, Miyakoji and Yamakiya. Some participants indicated that their participation in the Dialogue meetings encouraged them to engage in local actions in various places in the Fukushima Prefecture and gave them confidence to interact with experts;

- the Dialogue meetings gradually built up a network among the participants to establish connections between communities that did not speak to each other or did not know the actions taken by others. This network is now active outside Dialogue meetings and is also at the initiative of joint endeavours between communities;

- the presence of ICRP as a neutral third party was acknowledged as instrumental to provide trusted independent advice, to encourage healthy discussion, to make a link with experts in Japan and beyond, and to give more weight to residents' voices to the Prefectural authorities.

Finally, participants to this strategic meeting expressed a strong desire to continue the Dialogue meetings until all evacuees wishing to return to their homes could do so. They expressed the wish for the dialogues to directly engage a larger and more diverse group of participants, from and potentially beyond Fukushima Prefecture including participants from abroad.

\subsection{The creation of the Fukushima Dialogue NPO}

Building on the conclusions of the strategic meeting on the future of the Fukushima Dialogue organised on 13th February 18, a group of local residents established the Fukushima Dialogue Committee (FDC). The objective of this group was to organise the Fukushima Dialogue meeting planned for December 18 in Iwaki City and to create a Non-Profit Organisation (NPO) to ensure the continuity of the dialogue in the future.

Immediately following the Fukushima Dialogue 8 in Iwaki, the FDC organised a meeting to discuss how the dialogue should evolve to broaden the direct engagement of affected people in Fukushima and beyond and also increase the dissemination of its experiences and lessons within Japan and worldwide. This meeting was also devoted to the formal creation of the Fukushima Dialogue NPO, which held its first general assembly. The participants adopted the articles of the NPO constitution and elected R. Ando as President and O. Niwa and G. Kanno a farmer of Yamikiya as Vice Presidents.

Beyond the main objective to support the continuation of the organization of the Dialogue meetings, the NPO has also set the goal to manage and diffuse the material of the past Dialogue meetings and to establish partnerships with national and international organisations. The decision was also adopted to establish a web site of the NPO and to transfer all the material of the past Dialogue meetings on this new site.

\section{Some key lessons from the Dialogue meetings for nuclear post-accident management}

\subsection{The human dimension and the quality of living conditions}

The Dialogue meetings reemphasized that the irruption of radioactivity in the daily life of people is a significant rupture, which creates an unprecedented situation and deeply upsets the well-being of individuals and the quality of the living together of the local communities. This creates a complex situation generating a lot of questions, concerns and fears among the affected population (those living in contaminated areas as well as evacuees).

The management of the accident itself aiming at improving the radiological situation (i.e., the decontamination programme, interdictions and restrictions on economic and social activities in affected areas, controls of foodstuffs, the enforcement of radiological criteria but also the compensation scheme for the victims) introduces societal repercussions on the organisation of daily life and may severely disrupt human relationships.

The testimonies of participants to the Dialogue meetings confirmed the characteristics of the Chernobyl post-accident situation with regard to societal impact associated with the presence of radioactivity and the implementation of protective actions: the loss of control of inhabitants over their daily life, the disintegration of families, as well as the apprehension about the future, particularly that of children. People also expressed their fear to be progressively abandoned.

The Fukushima accident had a tremendous emotional and social impact that challenged lifestyle and also relationships within the local communities. In the aftermath of the accident, each individual was permanently confronted to the dilemma: to stay or to leave the contaminated territories and for evacuees to decide to return at home or not. Responses to this dilemma were largely depending on the general situation of the local communities as well as personal situation.

Through their testimonies and reflections, participants found the right words to better describe these human dimensions. They developed gradually a rich narrative based on their experiences helping them to engage in the rehabilitation process. This clearly confirms the importance in a post-nuclear accident situation of establishing places of dialogue involving the affected population and experts to develop the practical radiological protection culture favouring self-help protection, and to better understand and also be involved in the protection strategy implemented by authorities.

For the experts, these places of dialogue are the means, through listening to the affected people, to understand their concerns, their questions, and their expectations. For the affected people, it is not only a matter of obtaining general information about the situation of their community, but it contributes to improve their understanding on how they are personally exposed, and what to do to protect themselves and their communities.

The Dialogue meetings revealed the importance of considering carefully the specific characteristics of the affected communities while establishing the strategy for the rehabilitation of living conditions. The meetings held in different communities have shown that the same level of contamination can have different consequences depending on the local economic and societal characteristics, but also on the traditions, 
culture, and history of each community. This aspect calls for further reflection on the articulation of action at local, regional, and national levels in order to identify what types of governance mechanisms would be most suitable to consider this diversity.

Among the contributions of the Dialogue meetings, it is worth mentioning the protection of children. This was and remains a major concern for many parents in Fukushima, especially mothers. The post-accident experience of Fukushima and Chernobyl shows that the practical modalities of this protection depend not only on the local circumstances but also on the cultural contexts, and cover a wide spectrum of actions. Beyond differences, what is common is the desire not to keep children, and particularly teenagers, away from problems concerning their life in a contaminated territory. The testimonies from several adolescents made it clear that they intended to be involved in the future rehabilitation of the living conditions in the Fukushima Prefecture.

\subsection{The role of measurements}

The importance of a rapid characterisation of the radiological situation after a nuclear accident, adapted both to the needs of the public authorities (notably to guide decisions and actions) but also the affected people, is certainly one of the key lessons of the Dialogue meetings.

However, access to general information on average ambient dose rates of the areas and average contamination levels of food products is not sufficient for individuals to make decisions about their behaviours and activities. Only access to individual information on external and internal exposures allows people to link the radiological situation that characterises their familiar environment and their lifestyle. The Dialogue meetings reaffirmed the crucial role of individual measurements. On the one hand, they allow each individual to be able to know her/ his individual exposure and not only average values and to make informed decision. On the other hand, they allow the community to talk about the situation and to identify solutions together to improve the living conditions (Ando, 2016).

Several testimonies emphasized the interest for local communities of the distribution of individual dosimeters allowing to link the exposure to the activities of each individual to help people regaining control of the situation. In this perspective, it is obviously the responsibility of the public authorities to set up in the affected territories a system of individual monitoring of radiological exposures.

For the deployment of the monitoring strategy in postaccidental situation, the Dialogue meetings also pointed out the importance of clearly identifying the availability of equipment (including whole body counting, food monitoring devices, personal dosimeters...), their access for the different communities as well as the organisation of adequate training for the persons in charge of the monitoring. They emphasized the key role of places of dialogue to allow the interpretation of the results and the interaction between local communities and experts. The dissemination of the measurement results performed by public bodies and by citizens remains a sensitive issue, raising difficult problem related to the interpretation of the inevitable differences and the confidentiality aspects.

It is interesting to note that participants in the Dialogue meetings rarely referred to the dose criteria enforced by authorities to manage the radiological situation and never discussed their rationale. Discussions confirmed that people affected by the contamination were primarily motivated by what they can do to improve their situation from the radiological point of view. In turn, the dialogue participants discussed and analysed the impact of dose criteria on daily life, and particularly the fact that they may be a blocking factor for action and a source of division between people, with negative consequences for communities.

\subsection{The ethical position of the experts}

The Dialogue meetings also emphasized the key role of the empowerment of affected people through their direct engagement in the evaluation of the local situation, helping each individual to regain control on her/his radiological exposure and to restore her/his autonomy of decision, and freedom to make choices. In this context, some pitfalls should be avoided regarding people empowerment: trivialising the radiological risk, leaving people facing the risk alone, forcing people to make them staying in affected areas, engaging protective measures without involving affected people.

The ethical position of the expert in this context relies on the adoption of some key principles, which have been addressed during the dialogue seminars (Gariel et al., 2018):

- respect of the autonomy and freedom: experts should avoid taking decision for the people about their future. The decision to be made should respect the values and choices of each person. As mentioned above, these decisions involve many dimensions where the radiological issue is only one among others to be considered;

- commitment to be at the service of improving the protection and living conditions for the population (well-being): it is crucial for the experts involved in post-accidental situation to have a clear commitment to serve local communities. They should also promote and contribute to establish a good articulation between the different levels of decision (local, regional, national);

- adoption of a prudent attitude toward the radiological risk: experts should be consistent with the scientific knowledge related to radiation risk and the basic principles of radiological protection. They should recognize the limits of these knowledge as well as the various uncertainties associated with the management of post-accident situation. Their role is to well distinguish scientific facts and value judgments. Notably, it is never easy to conclude that the situation is safe. Several experts participating to the dialogue meetings acknowledged that one of the major difficulties they were facing is to talk about the effects and risks associated with exposure to ionizing radiation with the affected people.

\section{Concluding remarks}

The Fukushima Daiichi Nuclear Power Plant accident resulted in radiation becoming an unwelcome part of people's everyday life. This radiation, which cannot be seen or felt and is not something most people normally think of, inevitably raised concerns about possible health effects. As a result, people were confronted with a potential threat that is intangible, and against which they were fully unprepared to fight. For many, this very difficult situation created confusion and a lack of confidence. People's homeland, that they once loved and were proud of, transformed into something different. 
Everyday life was deteriorated, and ties between people and the community were impaired or broken. Under these conditions, for many, the only response is to avoid radiation as much as possible, until people's lives are choked by restrictions on what they can do and where they can go.

In this context, Dialogue meetings have played a key role for the participants to progressively understand the situation, to recover their capacity to take decision with regard to their life, and to contribute to the restoration of confidence in experts, and authorities. The Dialogue meetings helped the participants to identify margins of manoeuvre in their daily life, while respecting individual choices with regards to the decision to stay or not in their homeland. In several occasions, the Dialogue meeting allowed to establish connection between stakeholders, notably between producers and consumers, between neighbouring villages, between citizens and experts, even between Belarusian, Norwegian and Japanese citizens.

In total, approximately 1000 people participated directly in the Dialogue meetings after the Fukushima accident: many were local citizens, but many others were from elsewhere in Japan and the rest of the world. Many more people have been able to learn from the Dialogue meetings through social media, local media reporting, and information available through the ICRP and Ethos in Fukushima websites.

The presence of ICRP has been largely recognized by the participants as of great benefit for the two series of Dialogue meetings. The role of ICRP was crucial to be a neutral third party to provide trusted independent advice, be present as witness to encourage healthy discussion, make a link with experts in Japan and beyond, and lend weight to the proceedings so the voices are more likely to be heard beyond the Prefecture.

Among the main lessons for post-nuclear accident management, the Dialogue meetings emphasized that radiation protection is unavoidable but it cannot handle people's lives. It should be at the service of the well-being of individuals and the common good of the communities. It is notably important to develop monitoring strategy providing a focus on individual data and their distribution within the community to help people to characterize their own local radiological situation and to act accordingly. The Dialogue meetings also recalled the importance of engaging local professionals from education, health and administration in the development of practical radiological protection culture.

Finally, the Dialogue meetings highlighted the key role together with the difficulty, as in Belarus, of putting in place mechanisms for cooperation between the relevant actors (authorities, experts, professionals and the population) at local, regional and national levels, as well as the dissemination of good practices between community (Eikelmann and Hériard-Dubreuil, 2016). It is worth to note that the recently created Fukushima Dialogue NPO could significantly play this role in the future.

Acknowledgments. The authors would like first to sincerely thank all the Japanese and foreign participants to the Dialogue meetings for their active participation and the sincerity of their contributions. They especially thank the Belarusian and Norwegian participants for their fruitful testimonies. They also wish to thank all Japanese, foreign and international organisations that committed to the Dialogue meeting series.

The authors are very thankful to the organisations that have provided financial support to the Dialogue meetings: IRSN, NRPA, NEA-OECD, ASN, the Nippon Foundation and JAEA.

Finally, the Dialogue meetings would have not been possible without the commitment and support of their ICRP colleagues: C. Cousins, M. Kai, T. Homma, N. Ban, N. Hamada, M. Sasaki, H. Ogino and H. Fujita. May they receive here our warmest thanks.

\section{References}

Ando R. 2016. Measuring, discussing, and living together: Lessons from 4 years in Suetsugi. London: SAGE Publications; Ann. ICRP 45(1S): $75-83$.

Chiyoda Technol. 2018. Specifications of D-shuttle. Available from http://www.c-technol.co.jp/eng/e-dshuttle.

Eikelmann I, Hériard-Dubreuil G. 2016. Local populations facing long-term consequences of nuclear accidents: Lessons learnt from Fukushima and Chernobyl. Available from http://www.mutadis. org/publication-local-populations-facing-long-term-consequen ces-of-nuclear-accidents-lessons-learnt-from-fukushima-andtchernobyl-2/.

Gariel JC, Rollinger F, Schneider T. 2018. The role of experts in postaccident recovery: Lessons learnt from Chernobyl and Fukushima. In: Proceedings of the Fourth International Symposium on the System of Radiological Protection. Ann. ICRP 47(3/4).

Hériard Dubreuil G, Lochard J, Girard P, Guyonnet JF, Le Cardinal G, Lepicard S, Livolsi P, Monroy M, Ollagnon H, Pena-Vega A, Pupin V, Rigby J, Rolevitch I, Schneider T. 1999. Chernobyl post-accident management: The ETHOS project. Health. Phys. 77: 361-372.

ICRP. 2009a. Application of the Commission's Recommendations for the Protection of People in Emergency Exposure Situations. ICRP Publication 109. Ann. ICRP 39(1).

ICRP. 2009b. Application of the Commission's Recommendations to the Protection of People Living in Long-term Contaminated Areas After a Nuclear Accident or a Radiation Emergency. ICRP Publication 111. Ann. ICRP 39(3).

ICRP. 2016. Proceedings of the International Workshop on the Fukushima Dialogue Initiative. Ann. ICRP 45(2S).

Kotoba. 2015. Dialogues in Fukushima. The story of four years of dialogue for the rehabilitation of living conditions in the areas contaminated by the Fukushima accident. Web documentary, available from http://www.fukushima-dialogues.com.

Liland A, Skuterud L. 2013. Lessons learned from the Chernobyl accident in Norway. In: Social and Ethical Aspects of Radiation Risk Management. Radioactivity in the Environment (D. Oughton, S.O. Hansson, Eds.), Vol. 9, pp. 157-176. Oxford: Elsevier Science.

Lochard J. 2013. Stakeholder Engagement in Regaining Decent Living Conditions after Chernobyl. In: Social and Ethical Aspects of Radiation Risk Management. Radioactivity in the Environment (D. Oughto, S.O. Hansson, Eds.), Vol. 9, pp. 311-331. Elsevier.

Lochard J. 2016. The genesis of the ICRP Dialogue Initiative. Guest editorial. Proceedings of the International Workshop on the Fukushima Dialogue Initiative-Rehabilitation of Living Conditions after the Nuclear Accident. Date City Silk Hall, Fukushima Prefecture, Japan 12-13 December 2015. Ann. ICRP 45(2S): 7-13. 